\title{
Research Article \\ Second-Order Consensus for Multiagent Systems under Directed and Switching Topologies
}

\author{
Lixin Gao, Jingjing Zhang, and Wenhai Chen \\ Institute of Intelligent Systems and Decision, Wenzhou University, Zhejiang 325027, China \\ Correspondence should be addressed to Lixin Gao, gao-lixin@163.com
}

Received 24 June 2011; Accepted 25 August 2011

Academic Editor: Zidong Wang

Copyright (c) 2012 Lixin Gao et al. This is an open access article distributed under the Creative Commons Attribution License, which permits unrestricted use, distribution, and reproduction in any medium, provided the original work is properly cited.

We consider multiagent consensus problems in a decentralized fashion. The interconnection topology among the agents is switching and directed. The agent dynamics is expressed in the form of a double-integrator model. Two different cases are considered: one is the leaderfollowing case and the other is the leaderless case. Based on graph theory and the common Lyapunov function method, some sufficient conditions are established for the consensus stability of the considered systems with the neighbor-based feedback laws in both leader-following case and leaderless case, respectively. As special cases, the consensus conditions for balanced and undirected interconnection topology cases can be established directly. Finally, two numerical examples are given to illustrate the obtained results.

\section{Introduction}

In recent years, the coordination problem of multiple autonomous agents has drawn an increasing attention. It is because there are many applications of multiagent systems in many areas including cooperative control of unmanned air vehicles, flocking of birds, schooling for underwater vehicles, distributed sensor networks, attitude alignment for cluster of satellites, collective motion of particles, and distributed computations [1-5].

Although these practical backgrounds are different, the fundamental principles in the coordination of multiple spacecraft, robots, and even animals are very similar, that is, coordinating multiple agents to achieve a goal of the whole system by local information. As a result, a critical problem for coordinated control is to design appropriate protocols and algorithms such that the group of agents can reach consensus on the shared information in the presence of limited and unreliable information exchange and dynamically changing interaction topologies [6]. In [5], the author also pointed out that a key technology in cooperative control is the problem of consensus. The investigated feedback scheme associated 
with those problems is inspired by the aggregates of individuals in nature. Reynolds first proposed a computer animation model to simulate collective behaviors of multiple agents in [2]. In the fields of system and control, the development of consensus theory is primarily impelled by Vicsek's particle swarm model mentioned in [3]. In [1], Jadbabaie et al. gave a theoretical explanation of the consensus behavior of Vicsek's model and proved that the states of all the jointly connected agents converged to the same value or the value of a given leader. Ren and Beard [6] extended the work of Jadbabaie et al. [1] to the case of directed graphs and explored the minimum requirements to reach global consensus. Olfati-Saber and Murray studied the average-consensus problem with strongly connected and balanced directed topologies in [7], which is essentially the same problem as in [1,6] for continuoustime consensus scheme. Similar or generalized consensus problems have also been studied in [8-12]. There are also many interesting results about the similar topic for stochastic complex networks [13-15].

In real applications, the interacting topology between agents may change dynamically since the communication links between agents may be unreliable due to disturbances and/or subject to communication time delay. It is well known that switching of the communication topology and communication time delays may lower the system performance and even cause the network system to diverge or oscillate. Theoretically, the consensus in undirected switching topology is much easier than that of directed switching topology. Recently, some preliminary results have been reported to deal with the directed switching topology $[16,17]$.

Furthermore, with many practical applications, especially involving mechanical systems, the dynamics of agents is usually modeled as a double integrator $\ddot{x}=u$. There are many interesting agent-related works, such as in [18-20], involving double-integrator dynamics under switching and undirected interconnection topology among the agents. Most existing results are obtained under undirected interconnection topology. In directed topology case, [21] discussed this topic under the simple fixed topology. In [9], the authors pointed out that the system matrix related to this multiagent system with double-integrator dynamics does not satisfy the properties related to stochastic matrix and the norm-based method proposed by [1] may fail for the double-integrator form agent dynamic. Therefore, the Lyapunov-based approach is often chosen to solve consensus problem of multi-agent systems with double integrator model.

Motivated by the above works, we study a group of agents with the double-integrator dynamics. The main purpose of this paper is to develop a decentralized control strategy to reach the global consensus of the multi-agent systems under directed switching interconnection topology among the agents. Two different cases are considered: one is the leader-following case and the other is the leaderless case. First, we establish a sufficient consensus condition by constructing a parameter-dependent common Lyapunov function in leader-following formulation. The established consensus condition is expressed as a reducedorder Lyapunov matrix inequalities. As commonly known, it is not an easy task to construct a common Lyapunov function for a switching system, let alone the parameter-dependent common Lyapunov function. As for the leaderless case, the common Lyapunov method cannot be used directly. By using the Schur orthogonal transformation, we decomposes the group tracking dynamics into two subsystems: one represses the leader and the other represses a leader-following subsystem. Then, a sufficient condition is established by applying the obtained leader-following consensus condition to leader-following subsystem. As special cases, we can obtain consensus conditions directly for the multi-agent system with undirected and balanced switching interconnection topology. The jointly connected convergence condition can guarantee that the multi-agent with linear dynamical model 
achieves consensus. But for double-integrator model, we construct a counterexample to show that the jointly connected condition may not guarantee that the multi-agent system achieves consensus, which implies that the proposed globally reachable convergence condition may be moderatable and acceptable. By using similar analysis method, we also can probe consensus problems of $[9,18,19]$ with switching and directed interconnection topology. Finally, the numerical examples also show that the established consensus condition is solvable.

The rest of the paper is organized as follows. In Section 2, we give a formulation of the coordination problem with help of graph theory. Then in Section 3, some results on the consensus stability are obtained for the multi-agent system with fixed and varying interconnection topologies in the leader-following case, while in Section 4, the leaderless case is studied. In Section 5, two simulation examples are presented to illustrate our theoretical results. Finally, concluding remarks with discussions of the future work are given in Section 6.

The notation of this paper is standard. $R$ is the real number set. $I$ is an identity matrix with compatible dimension. $A^{T}$ denotes as transpose of a matrix $A$. For symmetric matrices $A$ and $B, A>(\geq) B$ means $A-B$ is positive (semi-)definite. $\lambda(A)$ represents an eigenvalue of $A$. For symmetric matrices $A, \lambda_{\min }(A)$ and $\lambda_{\max }(A)$ represent the minimum and maximum eigenvalue of $A$, respectively. $\|\bullet\|$ denotes the Euclidean norm. $\otimes$ denotes the Kronecker product, which satisfies (1) $(A \otimes B)(C \otimes D)=(A C) \otimes(B D)$; (2) if $A \geq 0$ and $B \geq 0$, then $A \otimes B \geq 0$.

\section{Problem Formulation}

Stability analysis of the group of agents is based on several results of algebraic graph theory. In this section, we first introduce some basic concepts and notations in graph theory that will be used throughout this paper. More details are available in [22].

We $n$ by $\mathcal{G}=\{\mho, \varepsilon, A\}$ with an index set $\mathcal{O}=\{1,2, \ldots, n\}$, where $\mathcal{U}=\left\{v_{1}, v_{2}, \ldots, v_{n}\right\}$ is the set of vertices, $\varepsilon \subset \mathcal{V} \times V$ is the set of edges of the digraph, and each edge is denoted by $\left(v_{i}, v_{j}\right) \in \varepsilon, i, j \in \mathcal{O}$, and a weighted adjacency matrix $A=\left[a_{i j}\right]$ has nonnegative adjacency elements $a_{i j}$. Throughout this paper, we assume that all the graphs have no edges from a node to itself. A weighted graph is called undirected if for all $\left(v_{i}, v_{j}\right) \in \varepsilon \Rightarrow\left(v_{j}, v_{i}\right) \in \varepsilon$ and $a_{i j}=a_{j i}$. Otherwise, the graph is called a directed graph or digraph. The adjacency elements associated with the edges of the graph are positive, that is $a_{i j}>0 \Leftrightarrow\left(v_{i}, v_{j}\right) \in \varepsilon$. Obviously, we also have $a_{i j}=0 \Leftrightarrow\left(v_{i}, v_{j}\right) \notin \varepsilon$. A path from vertex $v_{i}$ to vertex $v_{j}$ is a sequence of distinct vertices starting with $v_{i}$ and ending with $v_{j}$ such that consecutive pair of vertices make an edge of digraph. If there is a path from one node $v_{i}$ to another node $v_{j}$, then $v_{j}$ is said to be reachable from $v_{i}$. If a node $v_{i}$ is reachable from every other node of the digraph, then it is said to be globally reachable. Obviously, every node of an undirected connected graph is globally reachable.

The relationships between $n$ agents can be conveniently described by a simple digraph $\mathcal{G}$. We denote the set of all neighbor vertices of vertex $v_{i}$ by $\mathcal{N}_{i}=\left\{j \mid\left(v_{i}, v_{j}\right) \in \varepsilon\right\}$. Neighboring relations reflect physical proximity between two agents, or the existence of a communication channel [6]. Its degree matrix $D=\operatorname{diag}\left\{d_{1}, \ldots, d_{n}\right\} \in R^{n \times n}$ is a diagonal matrix, where diagonal elements $d_{i}=\sum_{j \in \mathcal{N}_{i}} a_{i j}$ for $i=1, \ldots, n$. Then the Laplacian matrix of $\mathcal{G}$ is defined as

$$
L=D-A \text {. }
$$


The following lemma relates the Laplacian matrix with its directed graph, as well as supplying an algebraic characterization. It is well known that the Laplacian matrix captures many interesting properties of the graphs. The result of Lemma 2.1 can be found in [6].

Lemma 2.1. The weighted graph Laplacian matrix $L$ associated with graph $\mathcal{G}$ has at least one zero eigenvalue, and all of the nonzero eigenvalues are located on the open right half plane. Furthermore, $L$ has exactly one zero eigenvalue if and only if the directed graph $\mathcal{G}$ has a globally reachable node.

Moreover, for a digraph with a globally reachable node, the $n$-dimensional eigenvector associated with the single zero eigenvalue is the vector of ones, $1:=(1,1, \ldots, 1)^{T}$. For undirected weight graph $\mathcal{G}, L$ is symmetric, positive and semidefinite, and all eigenvalues of $L$ are real and nonnegative.

A weighted graph $\mathcal{G}=(\mathcal{U}, \varepsilon, A)$ is said to be balanced if

$$
\sum_{j=1}^{n} a_{i j}=\sum_{j=1}^{n} a_{j i}, \quad i=1,2, \ldots, n,
$$

Any undirected weighted graph is balanced. Furthermore, a weighted graph is balanced if and only if $\mathbf{1}^{T} L=0$ (see [7]).

Let $\widetilde{\varepsilon}$ be the set of reverse edges of $\mathcal{G}$ obtained by reversing the order of all the pairs in $\varepsilon$. The mirror of $\mathcal{G}$ is denoted by an undirected graph in the form $\widehat{\mathcal{G}}=(\mathcal{U}, \widehat{\varepsilon}, \widehat{A})$ with the same set of nodes of $\mathcal{G}$, the set of edges $\widehat{\varepsilon}=\varepsilon \cup \widetilde{\varepsilon}$, and the symmetric adjacency matrix $\widehat{A}=\left[\widehat{a}_{i j}\right]$ with elements $\widehat{a}_{i j}=\left(a_{i j}+a_{j i}\right) / 2$. The Laplacian matrix for mirror graph $\widehat{\mathcal{G}}$ is $L(\widehat{\mathcal{G}})=(1 / 2)(L(\mathcal{G})+$ $\left.L^{T}(\mathcal{G})\right)$ if and only if $\mathcal{G}$ is balanced graph [7].

In what follows, we mainly consider a graph $\overline{\mathcal{G}}$ associated with the system with $n$ agents (labeled by $v_{i}, i=1,2, \ldots, n$ ) and one leader (labeled by $v_{0}$ ). A simple and directed graph $\mathcal{G}_{\mathcal{G}}$ describing the topology relation of these $n$ followers, and $\overline{\mathcal{G}}$ contains graph $\mathcal{G}$ and $v_{0}$ with the directed edges from some agents to the leader describes the topology relation among all agents. In other words, we denote a weighted digraph of order $n+1$ by $\overline{\mathcal{G}}=\{\overline{\mathcal{U}}, \bar{\varepsilon}, \bar{A}\}$, where $\bar{\mho}=\left\{v_{0}, v_{1}, \ldots, v_{n}\right\}$, and the weighted matrix has the following form:

$$
\bar{A}=\left(\begin{array}{ll}
0 & 0 \\
b & A
\end{array}\right), \quad b=\left(b_{1}, b_{2}, \cdots, b_{n}\right)^{T} \in R^{n}, A=\left(a_{i j}\right) \in R^{n \times n} .
$$

The element $a_{i j}>0$ represents that agent $i$ is connected to agent $j$, and $b_{i}>0$ represents that agent $i$ is connected to leader. Meanwhile, $a_{i j}=0$ and $b_{i}=0$ represents that agent $i$ is not connected to agent $j$ and leader, respectively. The induced subgraph $\mathcal{G}=\{\mathcal{U}, \varepsilon, A\}$ of $\overline{\mathcal{G}}$ represses the interconnection topology among $n$ following agents.

For the multi-agent system under consideration, the relationships between neighbors can change over time and the interconnection topology may be dynamically changing. Let $t_{1}=0, t_{2}, t_{3}, \ldots$ be an infinite time sequence at which the interconnection graph of the considered multi-agent system switches. Usually, it is assumed that chattering does not occur; that is, there is a constant $\tau_{0}>0$, often called dwell time, with $t_{i+1}-t_{i} \geq \tau_{0}$, for all $i$. Moreover, we assume that only finite possible interconnection topologies can be switched. In the leaderless case, denote $\mathcal{S}=\left\{\mathcal{G}_{1}, \mathcal{G}_{2}, \ldots, \mathcal{G}_{\mathcal{N}}\right\}$ as a set of the graphs of all possible topologies, while in the leader-following case, denote $\overline{\mathcal{S}}=\left\{\overline{\mathcal{G}}_{1}, \overline{\mathcal{G}}_{2}, \ldots, \overline{\mathcal{G}}_{N_{0}}\right\}$ as a set of the 
graphs of all possible topologies. Denote $D=\{1,2, \ldots, N\}$ and $p_{0}=\left\{1,2, \ldots, N_{0}\right\}$ as index sets, respectively.

In this paper, we intend to coordinate $n$ mobile agents with each agent expressed in the form of a double integrator:

$$
\begin{aligned}
& \dot{r}_{i}=p_{i}, \quad i=1, \ldots, n, \\
& \dot{p}_{\mathrm{i}}=u_{i},
\end{aligned}
$$

where $r_{i} \in R^{m}$ is the position of agent $i, p_{i} \in R^{m}$ its velocity and $u_{i} \in R^{m}$ its control inputs. In the leader-following case, the dynamics of the leader is taken as

$$
\begin{gathered}
\dot{r}_{0}=p_{0} \\
\dot{p}_{0}=a_{0}(t),
\end{gathered}
$$

where $r_{0} \in R^{m}$ and $p_{0} \in R^{m}$ are the position and velocity vectors of the leader, respectively, and $a_{0}(t) \in R^{m}$ is a known input, which may be regarded as some given policy known to all the agents. Particularly, $a_{0}(t) \equiv 0$ expresses that the leader moves in constant velocity.

The $n$ mobile agents are said to achieve global consensus if for any given initial values $r_{i}(0)$ and $p_{i}(0)(i=1,2, \ldots, n), \lim _{t \rightarrow+\infty}\left(r_{i}(t)-r_{j}(t)\right)=0$ and $\lim _{t \rightarrow+\infty}\left(p_{i}(t)-p_{j}(t)\right)=0$ for any $i, j=1,2, \ldots, n$. Thus, in the leader-following case, the multi-agent system achieves global consensus if and only if $\lim _{t \rightarrow+\infty}\left(r_{i}(t)-r_{0}(t)\right)=0$ and $\lim _{t \rightarrow+\infty}\left(p_{i}(t)-p_{0}(t)\right)=0$ for any initial values.

The control law is said to beneighbor control law or local control law if all the control inputs $u_{i}$ only depend on the states of agent $i$ and its neighbors. We are interested in using neighbor control law to solve the consensus problem. To this end, the controller $u_{i}$ of agent $i$, regarded as node $i$ in a graph, requires state information from a subset of the agent's flockmates, called the neighbor set $\mathcal{N}_{i}$ and defined as above. There are two cases considered for multi-agent system as follows.

\section{Leader-Following Case}

For any $i \in \mathcal{O}$, take the local control law, which is neighbor-based feedback law as follows:

$$
\begin{aligned}
u_{i}= & a_{0}(t)-\kappa\left(\sum_{j \in \mathcal{N}_{i}(t)} a_{i j}(t)\left(r_{i}-r_{j}\right)+b_{i}(t)\left(r_{i}-r_{0}\right)\right) \\
& -\kappa \gamma\left(\sum_{j \in \mathcal{N}_{i}(t)} a_{i j}(t)\left(p_{i}-p_{j}\right)+b_{i}(t)\left(p_{i}-p_{0}\right)\right),
\end{aligned}
$$

where $\mathcal{\kappa}$ is considered as a "control" parameter, which is positive constant and will be determined later, and $\gamma$ is a positive weighted parameter. The set of switching interconnection topology graph is assumed to be $\overline{\mathcal{G}}_{l}=\left\{\overline{\mathcal{V}}_{,}, \bar{\varepsilon}_{l}, \bar{A}_{l}\right\}, l \in \mathcal{D}_{0}$, where the $\bar{A}_{l}$ has the following form:

$$
\bar{A}_{l}=\left(\begin{array}{cc}
0 & 0 \\
b_{l} & A_{l}
\end{array}\right), \quad b_{l}=\left(b_{1}^{(l)}, b_{2}^{(l)}, \ldots, b_{n}^{(l)}\right)^{T} \in R^{n}, A_{l}=\left(a_{i j}^{(l)}\right) \in R^{n \times n} .
$$


In time $t$, the interconnection topology graph is assumed to be switched to $\overline{\mathcal{G}}_{l}, l \in p_{0}$, so $a_{i j}(t)$ and $b_{i}(t)$ are chosen by $a_{i j}(t)=a_{i j}^{(l)}$ and $b_{i}(t)=b_{i}^{(l)}$.

Denote $s_{j}=r_{j}-r_{0}$ and $q_{j}=p_{j}-p_{0}$. Take $s:=\left(s_{1}^{T}, \ldots, s_{n}^{T}\right)^{T} \in R^{m n}, q:=\left(q_{1}^{T}, \ldots, q_{n}^{T}\right)^{T} \in$ $R^{m n}$, and $u:=\left(u_{1}^{T}-a_{0}^{T}, u_{2}^{T}-a_{0}^{T}, \ldots, u_{n}^{T}-a_{0}^{T}\right)^{T} \in R^{m n}$. Then error dynamics of closed system (2.4)-(2.6) can be rewritten as

$$
\begin{gathered}
\dot{s}=q, \\
\dot{q}=u_{\sigma}=-\kappa\left(H_{\sigma} \otimes I_{m}\right) s-\kappa \gamma\left(H_{\sigma} \otimes I_{m}\right) q,
\end{gathered}
$$

where $H_{\sigma}=L_{\sigma}+B_{\sigma}, \sigma:[0,+\infty) \rightarrow p_{0}=\left\{1,2, \ldots, N_{0}\right\}$ is a piecewise constant switching signal with successive times when the topology of graph $\overline{\mathcal{G}}_{\text {switches. }} L_{\sigma}$ is the Laplacian matrix of the switching graph $\mathcal{G}_{1}$ consisting of $n$ vertices (presenting agents $1, \ldots, n$ ), and $B_{\sigma}$ is a diagonal matrix whose $i$ diagonal entry is $b_{i}(t)$ at time $t$.

\section{Leaderless Case}

Because there is no leader dynamics (2.5), take the local control law for any $i \in \mathcal{O}$ as follows:

$$
u_{i}=-\kappa \sum_{j \in \mathcal{N}_{i}(t)} a_{i j}(t)\left(r_{i}-r_{j}\right)-\kappa \gamma \sum_{j \in \mathcal{N}_{i}(t)} a_{i j}(t)\left(p_{i}-p_{j}\right)
$$

Let $r:=\left(r_{1}^{T}, \ldots, r_{n}^{T}\right)^{T}$ and $p:=\left(p_{1}^{T}, \ldots, p_{n}^{T}\right)^{T}$. Then we can rewrite the closed-loop system (2.4) and (2.9) as

$$
\begin{gathered}
\dot{r}=p, \\
\dot{p}=u_{\sigma}=-\kappa\left(L_{\sigma} \otimes I_{m}\right) r-\kappa \gamma\left(L_{\sigma} \otimes I_{m}\right) p,
\end{gathered}
$$

where $\sigma:[0,+\infty) \rightarrow D=\{1,2, \ldots, N\}$ is a piecewise constant switching signal to describe the switch of the interconnection graph $\mathcal{G}$. The $a_{i j}(t)$ is chosen by $a_{i j}(t)=a_{i j}^{(l)}, l \in \mathcal{D}$.

The next lemma shows well-known results and will be used later, which is given in [23].

Lemma 2.2. Suppose that a symmetric matrix is partitioned as

$$
\left(\begin{array}{ll}
C_{1} & C_{2} \\
C_{2}^{T} & C_{3}
\end{array}\right)
$$

where $C_{1}$ and $C_{3}$ are square. This matrix is positive definite if and only if $C_{1}$ is positive definite and $C_{3}-C_{2}^{T} C_{1}^{-1} C_{2}$ is positive definite.

In what follows, we first focus on the analysis of the leader-following case and then study the leaderless case in a similar trace. 


\section{Leader-Following Case}

In this section, we concentrate on the analysis of the multi-agent systems with a leader. If the information of the input $a_{0}(t)$ can be used in local control design, then we can prove that although the leader keeps changing, the agents can follow the leader that is, consensus is achieved.

Using neighbor-based feedback law (2.6), the error dynamics system (2.8) will be expressed in a compact form as follows:

$$
\dot{x}=\left(F_{\sigma} \otimes I_{m}\right) x,
$$

where

$$
x=\left(\begin{array}{l}
s \\
q
\end{array}\right), \quad F_{\sigma}=\left(\begin{array}{cc}
0 & I \\
-\kappa H_{\sigma} & -\kappa \gamma H_{\sigma}
\end{array}\right) \text {. }
$$

The interconnection topology graph is $\overline{\mathcal{G}}_{\sigma}(t)$, and the interconnection graphs associated with all followers are $\mathcal{G}_{\sigma}(t)$.

Note that $F_{\sigma}$ is not directly related to a stochastic matrix, and therefore, the method reported in [1] cannot be applied directly. In what follows, we will propose an approach based on common Lyapunov function for the system (3.1) to demonstrate the convergence of the dynamics system (3.1).

Now we give the main result in the leader-following case as follows.

Theorem 3.1. Suppose that the interconnection graph is connected for any interval $\left[t_{j}, t_{j+1}\right)$. If there exist a positive definite matrix $\bar{P}_{1}$ and a positive constant $\mu$ such that

$$
H_{l}^{T} \bar{P}_{1}+\bar{P}_{1} H_{l} \geq \mu I, \quad l \in p_{0}
$$

and taking a constant

$$
\kappa>\frac{2}{\mu \gamma^{2}} \lambda_{\max }\left(\bar{P}_{1}\right)
$$

then the local control law (2.6) can guarantee that multi-agent system (2.4), (2.5) achieves consensus for any given initial condition $r(0)$ and $p(0)$.

Proof. To prove the theorem, we consider the dynamics in each interval at first. Note that, in any interval (say $\left[t_{j}, t_{j+1}\right)$ ), the interconnection topology does not change. Therefore, $F_{\sigma(t)}$ is a constant matrix for $t \in\left[t_{j}, t_{j+1}\right)$ for any $j \geq 0$, and then the solution to (3.1) is well defined. Thus, we can assume that $\sigma(t)=l, l \in \mathcal{P}_{0}, t \in\left[t_{j}, t_{j+1}\right)$. Choose matrix $\widetilde{P}_{1}$ as

$$
\tilde{P}_{1}=\left(\begin{array}{cc}
2 \bar{P}_{1} & \gamma \bar{P}_{1} \\
r \bar{P}_{1} & \gamma^{2} \bar{P}_{1}
\end{array}\right)
$$


We can verify that $\widetilde{P}_{1}$ is positive definite by applying Lemma 2.2. Consider a common Lyapunov function $V(x)=x^{T}\left(\widetilde{P}_{1} \otimes I_{m}\right) x$, where $\widetilde{P}_{1}$ is defined in (3.5). Then, for any $l \in p_{0}$ which corresponds to interval $\left[t_{j}, t_{j+1}\right)$, we have

$$
\begin{aligned}
\left.\dot{V}(x)\right|_{(3.1)} & =x^{T}\left[\left(F_{l}^{T} \otimes I_{m}\right)\left(\widetilde{P}_{1} \otimes I_{m}\right)+\left(\tilde{P}_{1} \otimes I_{m}\right)\left(F_{l} \otimes I_{m}\right)\right] x \\
& =x^{T}\left[\left(F_{l}^{T} \widetilde{P}_{1}+\widetilde{P}_{1} F_{l}\right) \otimes I_{m}\right] x:=-x^{T}\left(Q_{l} \otimes I_{m}\right) x,
\end{aligned}
$$

where

$$
Q_{l}=\left(\begin{array}{cc}
\kappa \gamma\left(H_{l}^{T} \bar{P}_{1}+\bar{P}_{1} H_{l}\right) & \kappa \gamma^{2}\left(H_{l}^{T} \bar{P}_{1}+\bar{P}_{1} H_{l}\right)-2 \bar{P}_{1} \\
\kappa \gamma^{2}\left(H_{l}^{T} \bar{P}_{1}+\bar{P}_{1} H_{l}\right)-2 \bar{P}_{1} & \kappa \gamma^{3}\left(H_{l}^{T} \bar{P}_{1}+\bar{P}_{1} H_{l}\right)-2 \gamma \bar{P}_{1}
\end{array}\right)
$$

Note that

$$
\left(\begin{array}{cc}
\kappa \gamma & \kappa \gamma^{2} \\
\kappa \gamma^{2} & \kappa \gamma^{3}
\end{array}\right) \otimes\left(H_{l}^{T} \bar{P}_{1}+\bar{P}_{1} H_{l}-\mu I\right) \geq 0
$$

Then we have

$$
Q_{l} \geq Q:=\left(\begin{array}{cc}
\kappa \gamma \mu I & \kappa \gamma^{2} \mu I-2 \bar{P}_{1} \\
\kappa \gamma^{2} \mu I-2 \bar{P}_{1} & \kappa \gamma^{3} \mu I-2 \gamma \bar{P}_{1}
\end{array}\right)
$$

By using Lemma 2.2, (3.4) guarantees that matrix $Q$ is positive definite.

In addition, the maximum eigenvalue of $\widetilde{P}_{1}$ is

$$
\begin{gathered}
\lambda_{\max }\left(\widetilde{P}_{1}\right)=\frac{2+\gamma^{2}+2 \sqrt{1+\gamma^{4}}}{2} \lambda_{\max }\left(\bar{P}_{1}\right), \\
\min \frac{x^{T}\left(Q_{l} \otimes I\right) x}{x^{T}\left(\widetilde{P}_{1} \otimes I\right) x} \geq \frac{\lambda_{\min }(Q)}{\lambda_{\max }\left(\widetilde{P}_{1}\right)} .
\end{gathered}
$$

Let $\beta:=\lambda_{\min }(Q) / 2 \lambda_{\max }\left(\widetilde{P}_{2}\right)$. Then, we have $\dot{V}(x) \leq-2 \beta V(x)$, which implies $V(x) \leq$ $V(x(0)) e^{-2 \beta t}$. Moreover, we have

$$
\|x(t)\| \leq \frac{\sqrt{V(x)}}{\sqrt{\lambda_{\min }\left(\tilde{P}_{1}\right)}} \leq \frac{\sqrt{V(0)}}{\sqrt{\lambda_{\min }\left(\tilde{P}_{1}\right)}} e^{-\beta t} \leq \frac{\sqrt{\lambda_{\max }\left(\tilde{P}_{1}\right)}}{\sqrt{\lambda_{\min }\left(\tilde{P}_{1}\right)}}\|x(0)\| e^{-\beta t} .
$$

Thus we have $\lim _{t \rightarrow+\infty} x(t)=0$ with at least an exponent rate $\beta$. The proof is now completed. 
Remark 3.2. Obviously, the consensus problem of closed-loop multi-agent system (2.4), (2.5) and (2.6) is equivalent to stability problem of error system (3.1). The closed-loop multiagent system and the error system are both $2 n$-order systems, and the consensus condition (3.3) given in Theorem 3.1 is $n$-order Lyapunov matrix inequalities. Thus, the established consensus condition is a reduced order condition. In many applications, the system normally switches in finite model. The assumption that the system switches only in finite model is often used. Because the interconnection topology is modeled by weighted digraph, different weight will lead different interconnection topology. Although the set of nodes and the set of possible edges are finite, the set of possible interconnection topology may be infinite, but from the point of mathematics, the assumption that the index set $D$ is finite in Theorem 3.1 may be not necessary. From the proof of Theorem 3.1, we can also obtain the result of Theorem 3.1 by the assumption that condition (3.3) is satisfied for any element of an infinite index set $D$.

Note that the matrix $H=L+B$ plays a key role in the convergence analysis of system (3.1). A matrix is said to be a positive stable matrix if all its eigenvalues have positive real parts. The matrix $H=L+B$ is positive stable if and only if node $v_{0}$ is globally reachable in $\overline{\mathcal{G}}$ (see [18]).

Of course, the result of Theorem 3.1 can be applied to fixed topology case directly. Because $H$ is positive stable, there exists a positive definite matrix $\bar{P}_{2} \in R^{n \times n}$ such that

$$
H^{T} \bar{P}_{2}+\bar{P}_{2} H=I
$$

Therefore, we can obtain the following corollary for fixed topology case.

Corollary 3.3. Suppose the interconnection topology $\overline{\mathcal{G}}$ is fixed. If node $v_{0}$ is globally reachable in $\overline{\mathcal{G}}$ and the constant $\mathcal{\kappa}$ satisfies

$$
\kappa>\frac{2}{r^{2}} \lambda_{\max }\left(\bar{P}_{2}\right)
$$

then the local control law (2.6) can guarantee that system (2.4), (2.5) achieves consensus for any given initial condition $r(0)$ and $p(0)$.

Moreover, let $\lambda_{i}(H)=\alpha_{i}+j \beta_{i}(i=1,2, \ldots, n)$ be $i$ th eigenvalue of $H$. A necessary and sufficient condition for fixed topology case obtained by [21] is given as follows. The local control law (2.6) can guarantee that the multi-agent system in leader-following case achieves consensus for any given initial condition $r(0)$ and $p(0)$ if and only if node $v_{0}$ is globally reachable in $\overline{\mathcal{G}}$ and the constants $\mathcal{\kappa}$ and $\gamma$ satisfy

$$
\kappa>\frac{1}{\gamma^{2}} \max _{i} \frac{\beta_{i}^{2}}{\alpha_{i}\left[\alpha_{i}^{2}+\beta_{i}^{2}\right]} .
$$

The assumption that node $v_{0}$ is globally reachable in $\overline{\mathcal{G}}$ can guarantee that $\alpha_{i}>0$. For any given $\gamma>0, \alpha_{i}>0$, and $\beta_{i}$, it is not difficult to see (3.14) can be satisfied for larger enough $\kappa$. Obviously, (3.14) always holds for any $\gamma>0, \kappa>0$ if all $\beta_{i}=0$. When the interconnection graphs $\mathcal{G}_{\mathfrak{G}}$ associated with all followers are undirected and node $v_{0}$ is globally reachable in $\overline{\mathcal{G}}$, 
all eigenvalues of $H$ are real and positive (see [9]). Thus, in this fixed undirected case, multiagent system (2.4)-(2.6) achieves consensus for any given initial condition $r(0)$ and $p(0)$ if and only if node $v_{0}$ is globally reachable in $\overline{\mathcal{G}}$.

Consider the special case that the graph $\mathcal{G}_{\sigma}$ associated with all followers is balanced. The matrix $L_{\sigma}+L_{\sigma}^{T}$ is positive semidefinite. Moreover, we have (see [18]).

Lemma 3.4. Suppose $\mathcal{G}_{l}$ is balanced. Then $H_{l}^{T}+H_{l}$ is positive definite if and only if node $v_{0}$ is globally reachable in $\overline{\mathcal{G}_{l}}$.

Based on Lemma 3.4 and the fact that the set $p_{0}$ is finite, define

$$
\bar{\lambda}_{1}:=\frac{1}{2} \min _{l \in \mathcal{D}_{0}}\left\{\lambda_{\min }\left(H_{l}^{T}+H_{l}\right) \mid \text { node } 0 \text { is globally reachable of } \overline{\mathcal{G}}_{1} \text { and } \mathcal{G}_{l} \text { is balanced }\right\}>0
$$

which is fixed and depends directly on the constants $a_{i j}^{(l)}$ and $\beta_{i}^{(l)}$ for $i, j=1, \ldots, n$ and $l=1,2, \ldots, N$. Obviously, if all the interconnection graphs $\mathcal{G}_{\sigma}$ associated with followers are undirected and node $v_{0}$ is globally reachable in $\overline{\mathcal{G}}_{\sigma}, \bar{\lambda}_{1}$ can be expressed as $\bar{\lambda}_{1}=$ $\min _{p \in p_{0}}\left\{\lambda_{\min }\left(H_{l}\right)\right\}$. Now we propose following the corollary for the balanced graph case.

Corollary 3.5. In any interval $\left[t_{j}, t_{j+1}\right)$, suppose that node $v_{0}$ is globally reachable in the interconnection graph $\overline{\mathcal{G}}_{l}$, and $\mathcal{G}_{l}$ associated with all followers is balanced. Taking a constant

$$
\kappa>\frac{1}{r^{2} \bar{\lambda}_{1}}
$$

then multi-agent system (2.4)-(2.6) achieves consensus for any given initial condition $r(0)$ and $p(0)$.

Proof. According to Lemma 3.4 and the definition of $\bar{\lambda}_{1}$, we have

$$
H_{l}^{T} I+I H_{l} \geq 2 \bar{\lambda}_{1} I
$$

Then we can obtain the corollary directly by using the result of Theorem 3.1.

Because the interconnection graph considered in this paper is weighted graph, two graphs with same edge set and different weighted adjacency matrices are different interconnection graphs. Although the node set and edge are finite, the set of possible interconnection graph may be infinite. For the case that all the graphs $\mathcal{G}_{l}, l \in p_{0}$ are associated with all followers are balanced and the switching index set $p_{0}$ is infinite, we also can prove that multi-agent system achieves consensus by only assumption that there exists a positive constant $a_{\mathrm{min}}$ such that all nonzero weighted factors $a_{i j}^{(l)}, b_{i}^{(l)}$ are equal or greater than $a_{\min }$.

To probe the consensus stability condition in this case, let $\Gamma_{0}=\left\{\overline{\mathcal{G}}_{p}, p \in p_{0}\right\}$ denote the class of all possible interconnection graphs with the following properties: (1) $v_{0}$ is globally reachable node; (2) the interconnection graph $\mathcal{G}_{p}$ related to all followers is balanced; (3) all constant weighted factors $a_{i j}, b_{i}$ are equal or greater than a positive constant $a_{\text {min }} . p_{0}$ is index set of $\Gamma_{0}$, which is infinite set. Let $\widetilde{\Gamma}_{0}$ denote the class of all possible 
nonweighted interconnection graphs with the properties: (1) $v_{0}$ is globally reachable node; (2) the interconnection graph $\mathcal{G}$ related to all followers is undirected; (3) all nonzero weights are 1.

For any graph $\overline{\mathcal{G}} \in \Gamma_{0}$, we construct a related graph $\tilde{\mathcal{G}}$ by replacing all directed edges between all follower agents with undirected edges and all nonzero weights with 1 . Because $v_{0}$ is globally reachable node in the interconnection graph $\overline{\mathcal{G}}, v_{0}$ is also globally reachable node in $\widetilde{\mathcal{G}}$. Thus, we have $\widetilde{\mathcal{G}} \in \widetilde{\Gamma}_{0}$. Moreover, $H(\widetilde{\mathcal{G}})=B(\widetilde{\mathcal{G}})+L(\widetilde{\mathcal{G}})$ is symmetric and positive definite by Lemma 3.4. Because of the finiteness of node set and edge set, $\widetilde{\Gamma}_{0}$ is a finite set. Define

$$
\tilde{\lambda}_{1}:=\min _{\widetilde{\mathcal{G}}_{\in} \in \widetilde{\Gamma}_{0}}\left\{\lambda_{\min }(\widetilde{H}(\widetilde{\mathcal{G}}))\right\}
$$

which is fixed, and $\tilde{\lambda}_{1}>0$.

On the other hand, consider a weighted graph $\widehat{\mathcal{G}}$ with same node set and edge set as $\tilde{\mathcal{G}}_{l}$. In addition, its weight of edge $(i, j)$ is taken as $\left(a_{i j}+a_{j i}\right) / 2-a_{\min } \geq 0$ and weight of edge $(0, i)$ is taken as $2 b_{i}-a_{\min } \geq 0$. It is not difficult to see that

$$
\begin{aligned}
H(\widehat{\mathcal{G}}) & =B(\widehat{\mathcal{G}})+L(\widehat{\mathcal{G}}) \\
& =2 B(\overline{\mathcal{G}})+L(\overline{\mathcal{G}})+L^{T}(\overline{\mathcal{G}})-a_{\min }[B(\tilde{\mathcal{G}})+L(\tilde{\mathcal{G}})] \\
& =H(\overline{\mathcal{G}})+H^{T}(\overline{\mathcal{G}})-a_{\min } H(\tilde{\mathcal{G}}) .
\end{aligned}
$$

Noticing that $H(\widehat{\mathcal{G}})$ is symmetric and positive semidefinite matrix, we have

$$
H(\overline{\mathcal{G}})+H^{T}(\overline{\mathcal{G}}) \geq a_{\min } H(\tilde{\mathcal{G}}) \geq a_{\min } \tilde{\mathcal{I}}_{1} I
$$

Similarly, we can get the following result.

Corollary 3.6. Suppose that the interconnection graph $\overline{\mathcal{G}}_{\sigma}(t)$ of any interval $\left[t_{j}, t_{j+1}\right)$ belongs in $\Gamma_{0}$. Take a constant

$$
\kappa>\frac{2}{a_{\min } \gamma^{2} \tilde{\lambda}_{1}}
$$

Then the local control law (2.6) can guarantee that the multi-agent system in leader-following case achieves consensus for any given initial condition $r(0)$ and $p(0)$. 


\section{Leaderless Case}

In this section, similar to the analysis given in the last section, we probe the consensus problem for leaderless case. The involved interconnection graph is $\mathcal{G}$ instead of $\overline{\mathcal{G}}$. The closedloop system (2.10) will be expressed in a compact form as follows:

$$
\dot{x}=\left(F_{\sigma} \otimes I_{m}\right) x
$$

with

$$
x=\left(\begin{array}{l}
r \\
p
\end{array}\right), \quad F_{\sigma}=\left(\begin{array}{cc}
0 & I \\
-\kappa L_{\sigma} & -\kappa \gamma L_{\sigma}
\end{array}\right) .
$$

Noticing that vector $\mathbf{1}$ is the eigenvector of the Laplacian matrix $L_{\sigma}$ corresponding to its zero eigenvalue by Lemma 2.1, we choose an orthogonal matrix with form

$$
U=\left(\begin{array}{cccc}
\frac{1}{\sqrt{n}} & * & \cdots & * \\
\frac{1}{\sqrt{n}} & * & \cdots & * \\
\vdots & \vdots & \vdots & \vdots \\
\frac{1}{\sqrt{n}} & * & \cdots & *
\end{array}\right)
$$

where $*$ denotes the other entries in this matrix. We have

$$
U^{T} L_{\sigma} U=\left(\begin{array}{ll}
0 & \bar{B}_{\sigma} \\
0 & \bar{L}_{\sigma}
\end{array}\right),
$$

where $\bar{B}_{\sigma} \in R^{1 \times(n-1)}, \bar{L}_{\sigma} \in R^{(n-1) \times(n-1)}$. Taking $\bar{s}=U^{T} r$ and $\bar{q}=U^{T} p$, system (4.1) is equivalent to be described by

$$
\begin{gathered}
\dot{\bar{s}}=\bar{q} \\
\dot{\bar{q}}=\left[\left(\begin{array}{cc}
0 & -\kappa \bar{B}_{\sigma} \\
0 & -\kappa \bar{L}_{\sigma}
\end{array}\right) \otimes I_{m}\right] \bar{s}+\left[\left(\begin{array}{cc}
0 & -\kappa \gamma \bar{B}_{\sigma} \\
0 & -\kappa \gamma \bar{L}_{\sigma}
\end{array}\right) \otimes I_{m}\right] \bar{q} .
\end{gathered}
$$

For convenience, set

$$
\begin{aligned}
& \xi_{0}=\bar{s}_{1}, \\
& \xi_{1}=\left(\bar{s}_{2}^{T}, \bar{s}_{3}^{T}, \ldots, \bar{s}_{n}^{T}\right)^{T},
\end{aligned}
$$




$$
\begin{aligned}
& \psi_{0}=\bar{q}_{1}, \\
& \psi_{1}=\left(\bar{q}_{2}^{T}, \bar{q}_{3}^{T}, \ldots, \bar{q}_{n}^{T}\right)^{T} .
\end{aligned}
$$

Then the whole system decomposes into two subsystems as follows: one is

$$
\begin{gathered}
\dot{\xi}_{1}=\psi_{1}, \\
\dot{\psi}_{1}=-\kappa\left(\bar{L}_{\sigma} \otimes I_{m}\right) \xi_{1}-\kappa \gamma\left(\bar{L}_{\sigma} \otimes I_{m}\right) \psi_{1},
\end{gathered}
$$

and the other one is

$$
\begin{gathered}
\dot{\xi}_{0}=\psi_{0} \\
\dot{\psi}_{0}=-\kappa\left(\bar{B}_{\sigma} \otimes I_{m}\right) \xi_{1}-\kappa \gamma\left(\bar{B}_{\sigma} \otimes I_{m}\right) \psi_{1} .
\end{gathered}
$$

Notice that all eigenvalues of the matrix $\bar{L}_{\sigma}$ are eigenvalues of $L_{\sigma}$ from (4.4), which means all of the nonzero eigenvalues of $\bar{L}_{\sigma}$ are located on the open right half plane by Lemma 2.1. If the graph has a globally reachable node, the rank of $\bar{L}_{\sigma}$ is $n-1$, and therefore all the eigenvalues of matrix $\bar{L}_{\sigma}$ are located on the open right half plane, which implies matrix $\bar{L}_{\sigma}$ is positive stable matrix. Now we give the main result in leaderless case as follows.

Theorem 4.1. Suppose that the interconnection graph has a globally reachable node for any interval $\left[t_{j}, t_{j+1}\right)$. If there exist a matrix $U_{1} \in R^{n \times(n-1)}$, a positive definite $\bar{P}_{3} \in R^{(n-1) \times(n-1)}$, and a positive constant $\mu$ such that

$$
\begin{gathered}
U_{1}^{T} \mathbf{1}=0, \\
U_{1}^{T} U_{1}=I_{n-1}, \\
\left(U_{1}^{T} L_{l} U_{1}\right)^{T} \bar{P}_{3}+\bar{P}_{3}\left(U_{1}^{T} L_{l} U_{1}\right) \geq \mu I, \quad l \in D,
\end{gathered}
$$

and taking a constant

$$
\kappa>\frac{2}{\mu \gamma^{2}} \lambda_{\max }\left(\bar{P}_{3}\right)
$$

then the local control law (2.9) can guarantee that the multi-agent system in leaderless case achieves consensus for any given initial condition $r(0)$ and $p(0)$.

Proof. Take $U=\left((1 / \sqrt{n}) \mathbf{1}, U_{1}\right)$. Due to $U_{1}^{T} \mathbf{1}=0$ and $U_{1}^{T} U_{1}=I_{n-1}$, it is easy to know that $U$ is an orthogonal matrix and satisfies (4.4). Because the interconnection graph $\mathcal{G}_{l}$ has a globally 
reachable node, $\bar{L}_{l}=U_{1}^{T} L_{l} U_{1}$ is positive stable matrix. Applying the result of Theorem 3.1 to system (4.7), we have

$$
\lim _{t \rightarrow \infty}\left(\begin{array}{l}
\xi_{1} \\
\psi_{1}
\end{array}\right)=0 .
$$

Noticing that $U^{T} \mathbf{1}=(\sqrt{n}, 0, \ldots, 0)^{T}$, we have

$$
\begin{gathered}
r(t)-\frac{1}{\sqrt{n}} \xi_{0}(t) \mathbf{1}=U\left(\bar{s}-\frac{1}{\sqrt{n}} \xi_{0}(t) U^{T} \mathbf{1}\right)=U\left(\begin{array}{c}
0 \\
\xi_{1}(t)
\end{array}\right) \longrightarrow 0 \quad(t \longrightarrow+\infty), \\
p(t)-\frac{1}{\sqrt{n}} \psi_{0}(t) \mathbf{1}=U\left(\bar{q}-\frac{1}{\sqrt{n}} \psi_{0}(t) U^{T} \mathbf{1}\right)=U\left(\begin{array}{c}
0 \\
\psi_{1}(t)
\end{array}\right) \longrightarrow 0 \quad(t \longrightarrow+\infty),
\end{gathered}
$$

which imply $\lim _{t \rightarrow+\infty}\left(r_{i}(t)-r_{j}(t)\right)=0$ and $\lim _{t \rightarrow+\infty}\left(p_{i}(t)-p_{j}(t)\right)=0$ for any $i, j=1,2, \ldots, n$. This completes the proof.

Similarly, consider the special case that all the interconnection graphs $\mathcal{G}_{\sigma}$ are balanced. Due to $\mathbf{1}^{T} L_{\sigma}=L_{\sigma} \mathbf{1}=0$ for balanced graph $\mathcal{G}_{\sigma}$, we have

$$
U^{T} L_{\sigma} U=\left(\begin{array}{cc}
0 & 0 \\
0 & \bar{L}_{\sigma}
\end{array}\right), \quad \bar{L}_{\sigma} \in R^{(n-1) \times(n-1)},
$$

where $U$ is orthogonal matrix having form (4.3). The next lemma is given for $\bar{L}_{\sigma}^{T}+\bar{L}_{\sigma}$.

Lemma 4.2. If $\mathcal{G}_{\sigma}$ is balanced and has a globally reachable node, then $\bar{L}_{\sigma}^{T}+\bar{L}_{\sigma}$ is positive definite.

Proof. $1 / 2\left(L_{\sigma}^{T}+L_{\sigma}\right)$ is the Laplacian matrix of the mirror graph of $\mathcal{G}_{\sigma}$. Because $\mathcal{G}_{\sigma}$ is balanced and has a globally reachable node, the mirror graph is undirected and connected. Therefor, $1 / 2\left(L_{\sigma}^{T}+L_{\sigma}\right)$ is positive semidefinite and its rank is $n-1$. From (4.13), we have $\bar{L}_{\sigma}^{T}+\bar{L}_{\sigma}$ is positive definite.

Based on Lemma 4.2 and the fact that the set $p$ is finite, define

$$
\overline{\mathcal{\lambda}}_{2}:=\min _{l \in \mathcal{D}}\left\{\frac{1}{2} \lambda_{\min }\left(\bar{L}_{l}^{T}+\bar{L}_{l}\right) \mid \mathcal{G}_{l} \text { is balanced and has a globally reachable node }\right\}>0 .
$$

$\bar{\lambda}_{2}$ is also well defined. From (4.13), $\bar{\lambda}_{2}$ is equivalent to be expressed as

$$
\overline{\mathcal{\lambda}}_{2}=\min _{l \in \mathcal{D}}\left\{\frac{1}{2} \lambda_{2}\left(L_{l}^{T}+L_{l}\right) \mid \mathcal{G}_{l} \text { is balanced and has a globally reachable node }\right\}>0,
$$


where $\lambda_{2}\left(L_{l}^{T}+L_{l}\right)$ is the second small eigenvalue of $L_{l}^{T}+L_{l}$. Moreover, if all the interconnection graphs $\mathcal{G}_{\sigma}$ associated with any time interval are undirected and connected, then we have $\bar{\lambda}_{2}=\min _{l \in P}\left\{\lambda_{2}\left(L_{l}\right)\right\}$. For balanced graph case, the following corollary is similarly obtained as in Corollary 3.5.

Corollary 4.3. In any interval $\left[t_{j}, t_{j+1}\right)$, suppose that the interconnection graph $\mathcal{G}_{\sigma}(t)$ is balanced and has a globally reachable node. Taking a constant

$$
\kappa>\frac{1}{r^{2} \bar{\lambda}_{2}},
$$

then the local control law (2.9) can guarantee the multi-agent system in leaderless case achieves consensus for any given initial condition $r(0)$ and $p(0)$.

Similarly, denote by $\Gamma=\left\{\mathcal{G}_{p}, p \in D\right\}$ the class of all possible interconnection graphs with the following properties: (1) the interconnection graph $\mathcal{G}_{p}$ is balanced and has a globally reachable node; (2) all constant weighted factors $a_{i j}$ are equal or greater than a positive constant $a_{\min } . P$ is index set of $\Gamma$, which is infinite set. Let $\tilde{\Gamma}$ denote the class of all possible nonweighted interconnection graphs with the properties that the graph is balanced and connected. Thus $\tilde{\Gamma}$ is a finite set. Define

$$
\tilde{\lambda}_{2}:=\min _{\tilde{\mathcal{G}} \in \tilde{\Gamma}}\left\{\lambda_{2}\left[L^{T}(\tilde{\mathcal{G}})+L(\tilde{\mathcal{G}})\right]\right\}
$$

which is fixed, and $\widetilde{\lambda}_{2}>0$. By applying similar analysis of leader-following case, we propose the following result directly.

Corollary 4.4. Suppose that any interconnection graph $\mathcal{G}_{\sigma}(t)$ associated with interval $\left[t_{j}, t_{j+1}\right)$ belongs in $\Gamma$. Take a constant

$$
\kappa>\frac{2}{a_{\min } \gamma^{2} \tilde{\lambda}_{2}}
$$

Then the local control law (2.6) can guarantee that the multi-agent system in leader-following case achieves consensus for any given initial condition $r(0)$ and $p(0)$.

Remark 4.5. We define the center of the multi-agent system as $\bar{r}=(1 / n) \sum_{i=1}^{n} r_{i}$. The velocity of the center is expressed as $\bar{p}=(1 / n) \sum_{i=1}^{n} p_{i}$. If all the interconnection graphs $\mathcal{G}_{\sigma}$ associated with any time interval are balanced, the dynamics of center is given by 


$$
\begin{aligned}
\dot{\bar{r}} & =\frac{1}{n} \sum_{i=1}^{n} \dot{x}_{i}=\frac{1}{n} \sum_{i=1}^{n} v_{i}=\bar{p}, \\
\dot{\bar{p}} & =\frac{1}{n} \sum_{i=1}^{n} \dot{p}_{i}=\frac{1}{n} \sum_{i=1}^{n} u_{i} \\
& =\frac{1}{n} \sum_{i=1}^{n}\left[-\kappa \sum_{j \in \mathcal{N}_{i}} a_{i j}\left(r_{i}-r_{j}\right)-\kappa \sum_{j \in \mathcal{N}_{i}} a_{i j}\left(p_{i}-p_{j}\right)\right]=0 .
\end{aligned}
$$

Then, we can get that

$$
\begin{gathered}
\bar{p}(t)=\bar{p}(0)=\frac{1}{n} \sum_{i=1}^{n} p_{i}(0)=\frac{1}{\sqrt{n}} \psi_{0}(t), \\
\bar{r}(t)=\bar{r}_{0}(0)+\bar{p}(0) t=\frac{1}{\sqrt{n}} \xi_{0}(t) .
\end{gathered}
$$

The center $\bar{r}$ of the closed-loop system (2.10) will move at a constant speed $(1 / n) \sum_{i=1}^{n} p_{i}(0)$, which is the average initial velocity of all agents. The position and velocity of every agent will tend to the position and velocity of center, respectively, which means that the multi-agent network achieves consensus.

Remark 4.6. If the dynamics of the agent is linear, that is, $\dot{r}=u_{i}$, and the interconnection graph is jointly connected, as pointed out in the local control law can guarantee the multiagent system achieves consensus $[1,8]$. But for double-integrator model, the jointly connected condition may not guarantee that the multi-agent system achieves consensus. We propose a counterexample as follows. Thus the assumption that the interconnection graph has a globally reachable node in many references and also in this paper may be moderatable and acceptable.

\section{Counterexample 1}

The multi-agent system contains two agents, labeled by agent 1 and agent 2 . Without loss of generality, we assume $m=1$. For any positive constant $\alpha$, let $t_{0}=0, t_{1}, t_{2}, \ldots$ be an infinite time sequence, which satisfies $t_{2 k+1}=t_{2 k}+1$ and $t_{2 k+2}=t_{2 k+1}+\left(1 / 2 \alpha^{2}\right)+\left(1 / 4 \alpha^{2}\right)\left(e^{2 \alpha}+e^{-2 \alpha}\right)$, $k=0,1,2, \ldots$. Suppose that the two agents are connected in time interval $\left[t_{2 k}, t_{2 k+1}\right)$ and not connected in time interval $\left[t_{2 k+1}, t_{2 k+2}\right)$. The dynamics of the two agents can be expressed as

$$
\begin{aligned}
& \dot{r}_{i}=p_{i}, \quad i=1,2 . \\
& \dot{p}_{i}=u_{i},
\end{aligned}
$$


Take $\kappa=2 \alpha, \gamma=\alpha$. All nonzero weighted elements of graph are taken as 1 . Applying the local law (2.9) to the multi-agent system, the control input $u_{i}$ has the following form:

$$
\begin{aligned}
& u_{1}(t)= \begin{cases}-2 \alpha\left(r_{1}-r_{2}\right)-2 \alpha^{2}\left(p_{1}-p_{2}\right), & t \in\left[t_{2 k}, t_{2 k+1}\right), \\
0, & t \in\left[t_{2 k+1}, t_{2 k+2}\right),\end{cases} \\
& u_{2}(t)= \begin{cases}-2 \alpha\left(r_{2}-r_{1}\right)-2 \alpha^{2}\left(p_{2}-p_{1}\right), & t \in\left[t_{2 k}, t_{2 k+1}\right), \\
0, & t \in\left[t_{2 k+1}, t_{2 k+2}\right) .\end{cases}
\end{aligned}
$$

The initial position and velocity of agents are taken as $r_{1}(0)=-r_{2}(0)=\left(e^{2 \alpha}-2 \alpha+1\right) / 4 \alpha^{2}$ and $p_{1}(0)=-p_{2}(0)=1$. From the symmetry of the input and initial values, we can know that $r_{1}=-r_{2}$ and $p_{1}=-p_{2}$. Then, we can obtain

$$
r_{1}(t)=-r_{2}(t)= \begin{cases}{\left[\frac{e^{2 \alpha}-2 \alpha+1}{4 \alpha^{2}}+\frac{e^{2 \alpha}+1}{2 \alpha}\left(t-t_{4 k}\right)\right] e^{-2 \alpha\left(t-t_{4 k}\right),}} & t \in\left[t_{4 k}, t_{4 k+1}\right), \\ -\left[\frac{e^{2 \alpha}-2 \alpha+1}{4 \alpha^{2}}+\frac{1}{4 \alpha^{2}} e^{-2 \alpha^{2}}-\left(t-t_{4 k+1}\right),\right. & t \in\left[t_{4 k+1}, t_{4 k+2}\right), \\ p_{1}(t)=-p_{2}(t)= \begin{cases}\left.\left.e^{2 \alpha}+1-t_{4 k}\right)\right] e^{-2 \alpha\left(t-t_{4 k}\right)}, & t \in\left[t_{4 k+2}, t_{4 k+3}\right), \\ -\frac{1+2 \alpha}{4 \alpha^{2}}-\frac{1}{4 \alpha^{2}} e^{-2 \alpha^{2}}+\left(t-t_{4 k+3}\right), & t \in\left[t_{4 k+3}, t_{4 k+4}\right), \\ -e^{-2 \alpha\left(t-t_{4 k}\right)}+\left(e^{2 \alpha}+1\right)\left(t-t_{4 k+2}\right) e^{-2 \alpha\left(t-t_{4 k+2}\right)}, & t \in\left[t_{4 k+2}, t_{4 k+3}\right), \\ 1, & t \in\left[t_{4 k+3}, t_{4 k+4}\right),\end{cases} \end{cases}
$$

for $k=0,1,2, \ldots$. Although the interconnection graph related with any time interval $\left[t_{2 k+1}, t_{2 k+2}\right)$ is not connected, the jointed graph related to the multi-agent system is jointly connected in any interval $\left[t_{2 k}, t_{2(k+1)}\right)$. Thus, the interconnection topology of the multi-agent system satisfies jointly connected condition proposed by [1]. On the other hand, it is easy to see that the multi-agent system cannot achieve consensus for any positive constant $\alpha$ in this case.

\section{Simulation Examples}

In this section, to illustrate our theoretical results derived in the above sections, we will provide two numerical simulations. Without loss of generality, we take $m=1$ in numerical simulation. In leader-follower case, consider a multi-agent system with one leader and six followers. The interconnection topology is arbitrarily switched with switching period 1 
among four graphs $\overline{\mathcal{G}}_{i}(i=1,2,3,4)$. The Laplacian matrices $L_{i}(i=1,2,3,4)$ for the four subgraphs $\mathcal{G}_{i}(i=1,2,3,4)$ are

$$
\begin{aligned}
L_{1} & =\left(\begin{array}{cccccc}
3.5 & -1.5 & 0 & 0 & 0 & -2 \\
-1 & 5.5 & -2.5 & 0 & -2 & 0 \\
0 & -1 & 2 & -1 & 0 & 0 \\
0 & 0 & -2 & 5 & -1 & -2 \\
0 & -2 & 0 & -1 & 5 & -2 \\
-1 & 0 & 0 & -1 & -2 & 4
\end{array}\right), \quad L_{2}=\left(\begin{array}{ccccccc}
5 & -1 & -2 & 0 & -2 & 0 \\
-1.5 & 4.5 & -1 & 0 & 0 & -2 \\
-2 & -1 & 4 & 0 & -1 & 0 \\
0 & 0 & 0 & 2 & 0 & -2 \\
-1 & 0 & -1 & 0 & 2 & 0 \\
0 & -2 & 0 & -2 & 0 & 4
\end{array}\right), \\
L_{3} & =\left(\begin{array}{cccccc}
3 & 0 & 0 & -2 & 0 & -1 \\
0 & 2 & 0 & -1 & -1 & 0 \\
0 & 0 & 2 & 0 & 0 & -2 \\
-2 & -1 & 0 & 5 & -2 & 0 \\
0 & -1 & 0 & -2 & 3 & 0 \\
-2 & 0 & -2 & 0 & 0 & 4
\end{array}\right), \quad L_{4}=\left(\begin{array}{cccccc}
2 & 0 & 0 & -1 & -1 & 0 \\
0 & 4 & -2 & 0 & -2 & 0 \\
0 & -2 & 5 & -2 & 0 & -1 \\
-2 & 0 & -2 & 4 & 0 & 0 \\
-1 & -2 & 0 & 0 & 5 & -2 \\
0 & 0 & -1 & 0 & -2 & 3
\end{array}\right)
\end{aligned}
$$

and the diagonal matrices for the interconnection relationship between the leader and the followers are

$$
\begin{array}{ll}
B_{1}=\operatorname{diag}(1,0,0,1,0,0), & B_{2}=\operatorname{diag}(0,1,0,1,0,0), \\
B_{3}=\operatorname{diag}(1,0,0,0,1,1), & B_{4}=\operatorname{diag}(0,0,1,1,0,0) .
\end{array}
$$

The positive definite

$$
\bar{P}_{2}=\left(\begin{array}{llllll}
4.60 & 0.58 & 0.18 & 0.65 & 0.78 & 0.67 \\
0.58 & 4.01 & 0.95 & 0.41 & 0.92 & 0.85 \\
0.18 & 0.95 & 6.12 & 0.74 & 0.80 & 1.38 \\
0.65 & 0.41 & 0.74 & 3.51 & 0.59 & 0.61 \\
0.78 & 0.92 & 0.80 & 0.59 & 5.08 & 1.05 \\
0.67 & 0.85 & 1.38 & 0.61 & 1.05 & 4.92
\end{array}\right)
$$

and $\mu=1$ satisfy condition (3.3). Take $\gamma=2.5$ and $\kappa=3>\left(2 / \mu \gamma^{2}\right) \lambda_{\max }\left(\bar{P}_{2}\right)$, where $\lambda_{\max }\left(\bar{P}_{2}\right)=$ 8.81 .

The initial positions and velocities of the all agents are randomly produced. The position and velocity errors in Figure 1 are defined as $r_{i}-r_{0}$ and $p_{i}-p_{0}$, respectively. Figure 1 shows that the follower agents can track the leader.

In leaderless case, consider a multi-agent system with six agents. The interconnection topology is also arbitrarily switched with switching period 1 among four graphs $\mathcal{G}_{i}(i=$ 


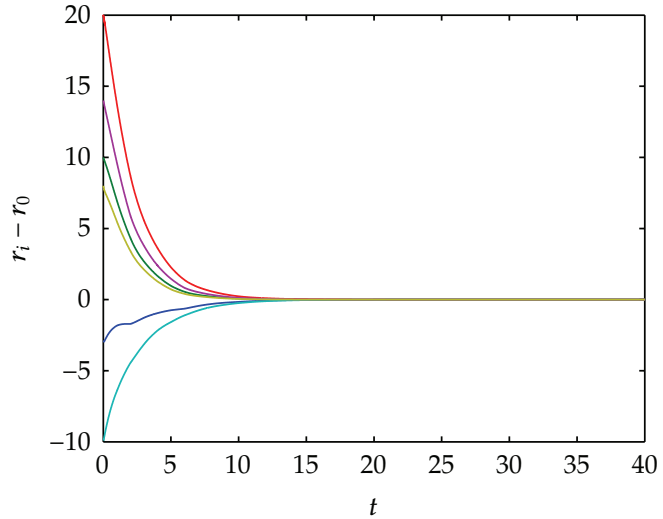

(a)

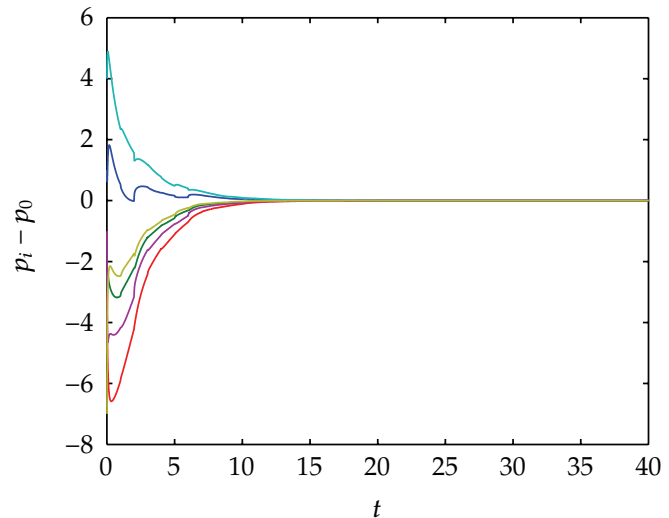

(b)

Figure 1: Position and velocity tracking errors of six followers.

$1,2,3,4)$. The Laplacian matrices $L_{i}(i=1,2,3,4)$ of graphs $\mathcal{G}_{i}$ are also defined as above. The matrix

$$
U_{1}=\left(\begin{array}{ccccc}
-0.4695 & 0.4926 & 0.3434 & 0.3596 & -0.3507 \\
-0.5804 & -0.5372 & -0.0859 & -0.4456 & -0.0433 \\
0.3598 & 0.3469 & -0.5239 & -0.3718 & -0.4133 \\
0.5426 & -0.2934 & 0.6559 & -0.0941 & -0.1174 \\
0.0109 & 0.3577 & 0.0222 & -0.1558 & 0.8249 \\
0.1366 & -0.3666 & -0.4118 & 0.7077 & 0.0998
\end{array}\right),
$$

the positive definite

$$
\bar{P}_{3}=\left(\begin{array}{ccccc}
0.7577 & -0.0131 & -0.0377 & 0.0021 & -0.0559 \\
-0.0131 & 0.6863 & 0.0262 & 0.0109 & -0.0595 \\
-0.0377 & 0.0262 & 0.8184 & 0.0813 & 0.0027 \\
0.0021 & 0.0109 & 0.0813 & 0.8299 & 0.0194 \\
-0.0559 & -0.0595 & 0.0027 & 0.0194 & 0.8698
\end{array}\right)
$$

and $\mu=1$ such that they satisfy condition (4.9). Take $\gamma=2.5$ and $\kappa=1>\left(2 / \mu \gamma^{2}\right) \lambda_{\max }\left(\bar{P}_{3}\right)$, where $\lambda_{\max }\left(\bar{P}_{3}\right)=0.93$. The position and velocity errors in Figure 2 are defined as $r_{i}-\bar{r}$ and $p_{i}-\bar{p}$, respectively. Figure 2 shows the multi-agent system in leaderless case can achieve consensus. 


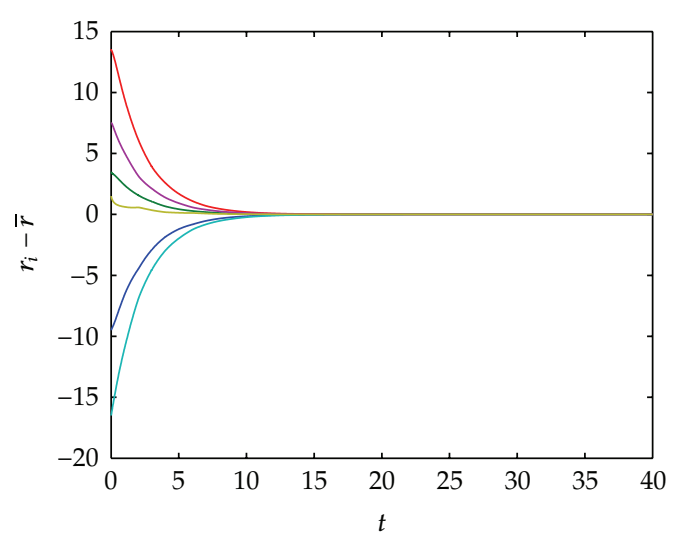

(a)

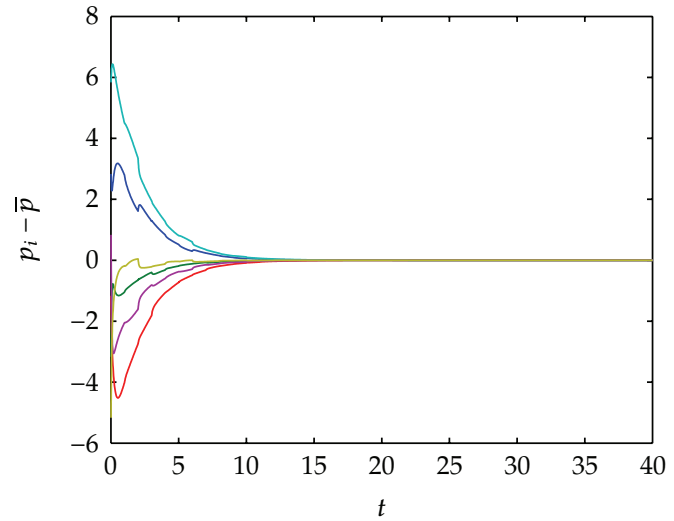

(b)

Figure 2: Position and velocity tracking errors of six followers.

\section{Conclusion}

In this paper, we have investigated the control of multi-agent systems with varying directed interconnection topologies using graph theory and stability theory. The proposed consensus strategy is neighbor-based law that is, each agent regulated its position and velocity based on its neighbor agents. There are two different cases considered in this study. One is the leader-following case and the other the leaderless case. To make this less conservative, a parameter-dependent common Lyapunov function (CLF) was constructed to analyze the stability of the closed system. Some sufficient conditions are given to achieve consensus of these mobile agents with the proposed local control strategies in both leader-following and leaderless cases, respectively. Of course, we can obtain consensus condition directly for the multi-agent system with the switching and undirected interconnection topology or directed balanced interconnection topology. Due to conservativeness of the common Lyapunov function method, we will probe less conservative method in our future work. We also will probe multi-agent $H_{\infty}$ consensus control problems with external disturbance under time delay directed switching topologies in our future work. Moreover, several numerical simulations were shown to verify the theoretical analysis.

\section{Acknowledgment}

This work was supported by the National Nature Science Function of China under Grants nos. 61074123, 60674071, and 61174063.

\section{References}

[1] A. Jadbabaie, J. Lin, and A. S. Morse, "Coordination of groups of mobile autonomous agents using nearest neighbor rules," IEEE Transactions on Automatic Control, vol. 48, no. 6, pp. 988-1001, 2003.

[2] C. W. Reynolds, "Flocks, herds, and schools: a distributed behavioral model," Computer Graphics, vol. 21, no. 4, pp. 25-34, 1987.

[3] T. Vicsek, A. Czirk, E. Ben-Jacob, I. Cohen, and O. Shochet, "Novel type of phase transition in a system of self-driven particles," Physical Review Letters, vol. 75, no. 6, pp. 1226-1229, 1995. 
[4] S. Mu, T. Chu, and L. Wang, "Coordinated collective motion in a motile particle group with a leader," Physica A, vol. 351, no. 2-4, pp. 211-226, 2005.

[5] R. M. Murray, "Recent research in cooperative control of multivehicle systems," Journal of Dynamic Systems, Measurement and Control, Transactions of the ASME, vol. 129, no. 5, pp. 571-583, 2007.

[6] W. Ren and R. W. Beard, "Consensus seeking in multiagent systems under dynamically changing interaction topologies," IEEE Transactions on Automatic Control, vol. 50, no. 5, pp. 655-661, 2005.

[7] R. Olfati-Saber and R. M. Murray, "Consensus problems in networks of agents with switching topology and time-delays," IEEE Transactions on Automatic Control, vol. 49, no. 9, pp. 1520-1533, 2004.

[8] L. Gao and D. Cheng, "Comment on coordination of groups of mobile autonomous agents using nearest neighbor rules," IEEE Transactions on Automatic Control, vol. 50, no. 11, pp. 1913-1916, 2005.

[9] Y. Hong, J. Hu, and L. Gao, "Tracking control for multi-agent consensus with an active leader and variable topology," Automatica, vol. 42, no. 7, pp. 1177-1182, 2006.

[10] F. Xiao and L. Wang, "Asynchronous consensus in continuous-time multi-agent systems with switching topology and time-varying delays," IEEE Transactions on Automatic Control, vol. 53, no. 8, pp. 1804-1816, 2008.

[11] B. Shen, Z. Wang, and Y. S. Hung, "Distributed $\mathrm{H}_{\infty}$-consensus filtering in sensor networks with multiple missing measurements: the finite-horizon case," Automatica, vol. 46, no. 10, pp. 1682-1688, 2010.

[12] W. Ni and D. Cheng, "Leader-following consensus of multi-agent systems under fixed and switching topologies," Systems and Control Letters, vol. 59, no. 3-4, pp. 209-217, 2010.

[13] J. Liang, Z. Wang, and X. Liu, "State estimation for coupled uncertain stochastic networks with missing measurements and time-varying delays: the discrete-time case," IEEE Transactions on Neural Networks, vol. 20, no. 5, pp. 781-793, 2009.

[14] Z. Wang, Y. Wang, and Y. Liu, "Global synchronization for discrete-time stochastic complex networks with randomly occurred nonlinearities and mixed time delays," IEEE Transactions on Neural Networks, vol. 21, no. 1, Article ID 5342442, pp. 11-25, 2010.

[15] B. Shen, Z. Wang, and X. Liu, "Bounded $\mathrm{H}_{\infty}$ synchronization and state estimation for discrete timevarying stochastic complex networks over a finite horizon," IEEE Transactions on Neural Networks, vol. 22, no. 1, pp. 145-157, 2011.

[16] P. Lin, Y. Jia, and L. Li, "Distributed robust $\mathrm{H}_{\infty}$ consensus control in directed networks of agents with time-delay," Systems and Control Letters, vol. 57, no. 8, pp. 643-653, 2008.

[17] Y. Sun, L. Wang, and G. Xie, "Average consensus in networks of dynamic agents with switching topologies and multiple time-varying delays," Systems and Control Letters, vol. 57, no. 2, pp. 175-183, 2008.

[18] J. Hu and Y. Hong, "Leader-following coordination of multi-agent systems with coupling time delays," Physica A, vol. 374, no. 2, pp. 853-863, 2007.

[19] Y. Hong, G. Chen, and L. Bushnell, "Distributed observers design for leader-following control of multi-agent networks," Automatica, vol. 44, no. 3, pp. 846-850, 2008.

[20] L. X. Gao, H. J. Yan, and D. Jin, "Consensus problems in multi-agent systems with double integrator model," Chinese Physics B, vol. 19, no. 5, Article ID 050520, 8 pages, 2010.

[21] W. Yu, G. Chen, and M. Cao, "Some necessary and sufficient conditions for second-order consensus in multi-agent dynamical systems," Automatica, vol. 46, no. 6, pp. 1089-1095, 2010.

[22] C. Godsil and G. Royle, Alegbraic Graph Theory, Springer, New York, NY, USA, 2001.

[23] R. Horn and C. Johnson, Matrix Analysis, Cambridge University Press, New York, NY, USA, 1985. 


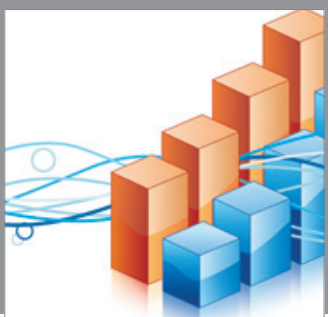

Advances in

Operations Research

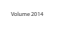

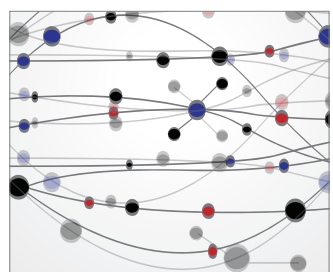

\section{The Scientific} World Journal
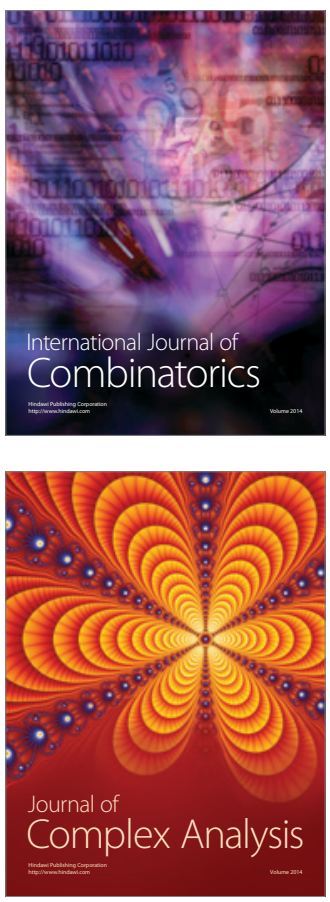

International Journal of

Mathematics and

Mathematical

Sciences
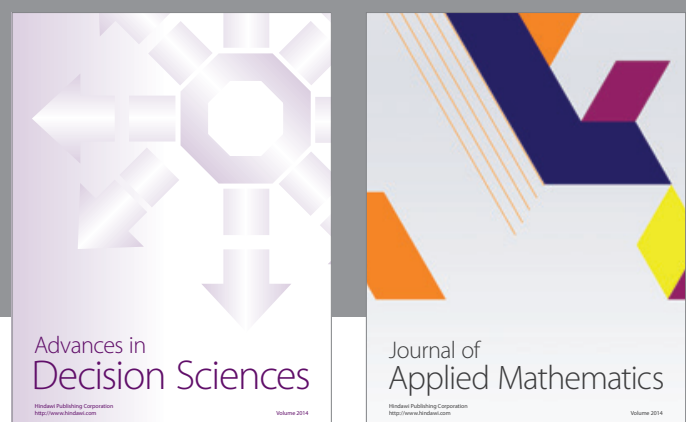

Journal of

Applied Mathematics
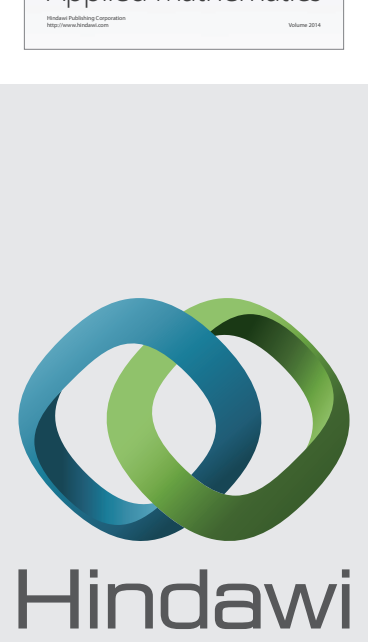

Submit your manuscripts at http://www.hindawi.com
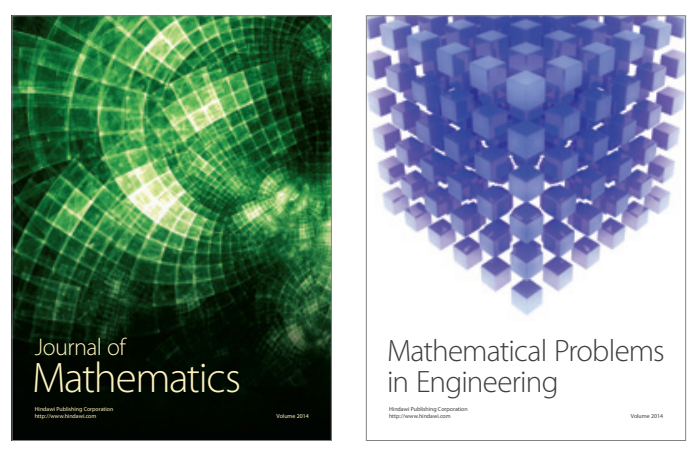

Mathematical Problems in Engineering
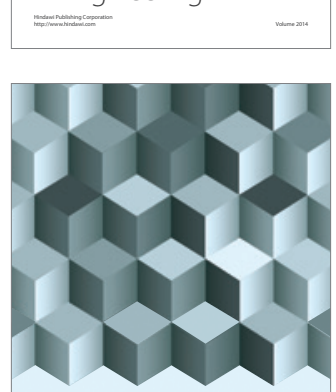

Journal of

Function Spaces
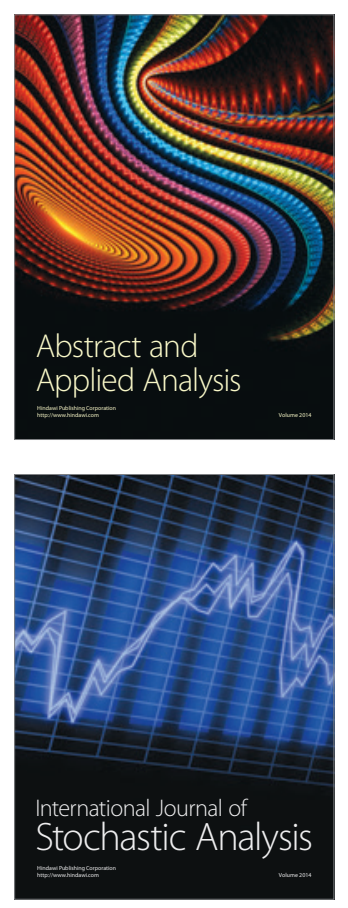

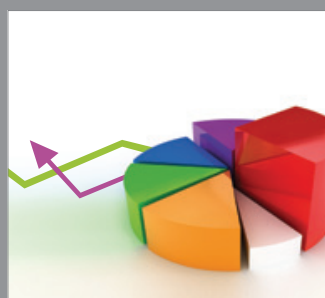

ournal of

Probability and Statistics

Promensencen
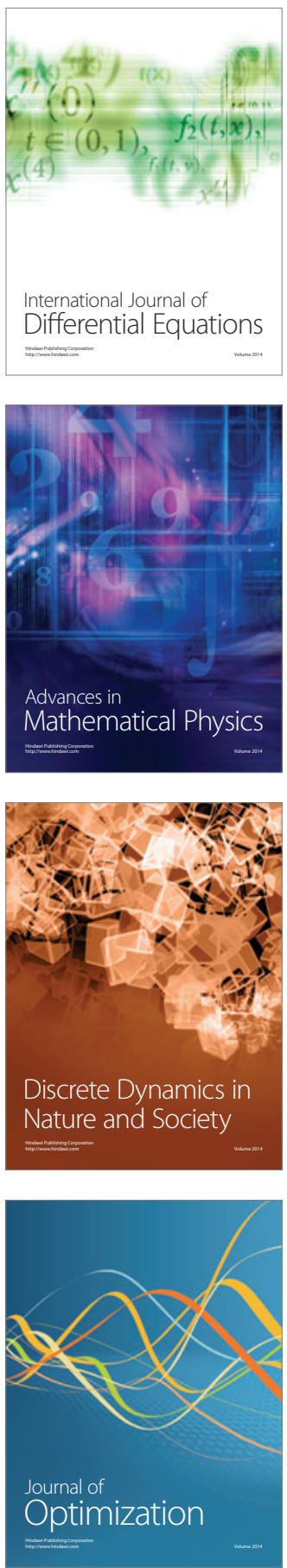